\section{$\underset{\text { hommes }}{\text { \& migrations }}$}

\section{Hommes \& migrations}

Revue française de référence sur les dynamiques

migratoires

$1304 \mid 2013$

Frontières

\title{
Les demandeurs d'asile subsahariens en Israël
}

De nouvelles frontières territoriales, politiques et sociales

\section{Lisa Anteby-Yemini}

\section{(2) OpenEdition}

12 Journals

\section{Édition électronique}

URL : http://journals.openedition.org/hommesmigrations/2645

DOI : 10.4000/hommesmigrations.2645

ISSN : 2262-3353

Éditeur

Musée national de l'histoire de l'immigration

\section{Édition imprimée}

Date de publication : 1 octobre 2013

Pagination : $67-74$

ISBN : 978-2-919040-24-7

ISSN : 1142-852X

Référence électronique

Lisa Anteby-Yemini, « Les demandeurs d'asile subsahariens en Israël », Hommes \& migrations [En ligne], 1304 | 2013, mis en ligne le 01 janvier 2017, consulté le 22 avril 2019. URL : http://

journals.openedition.org/hommesmigrations/2645; DOI : 10.4000/hommesmigrations.2645 


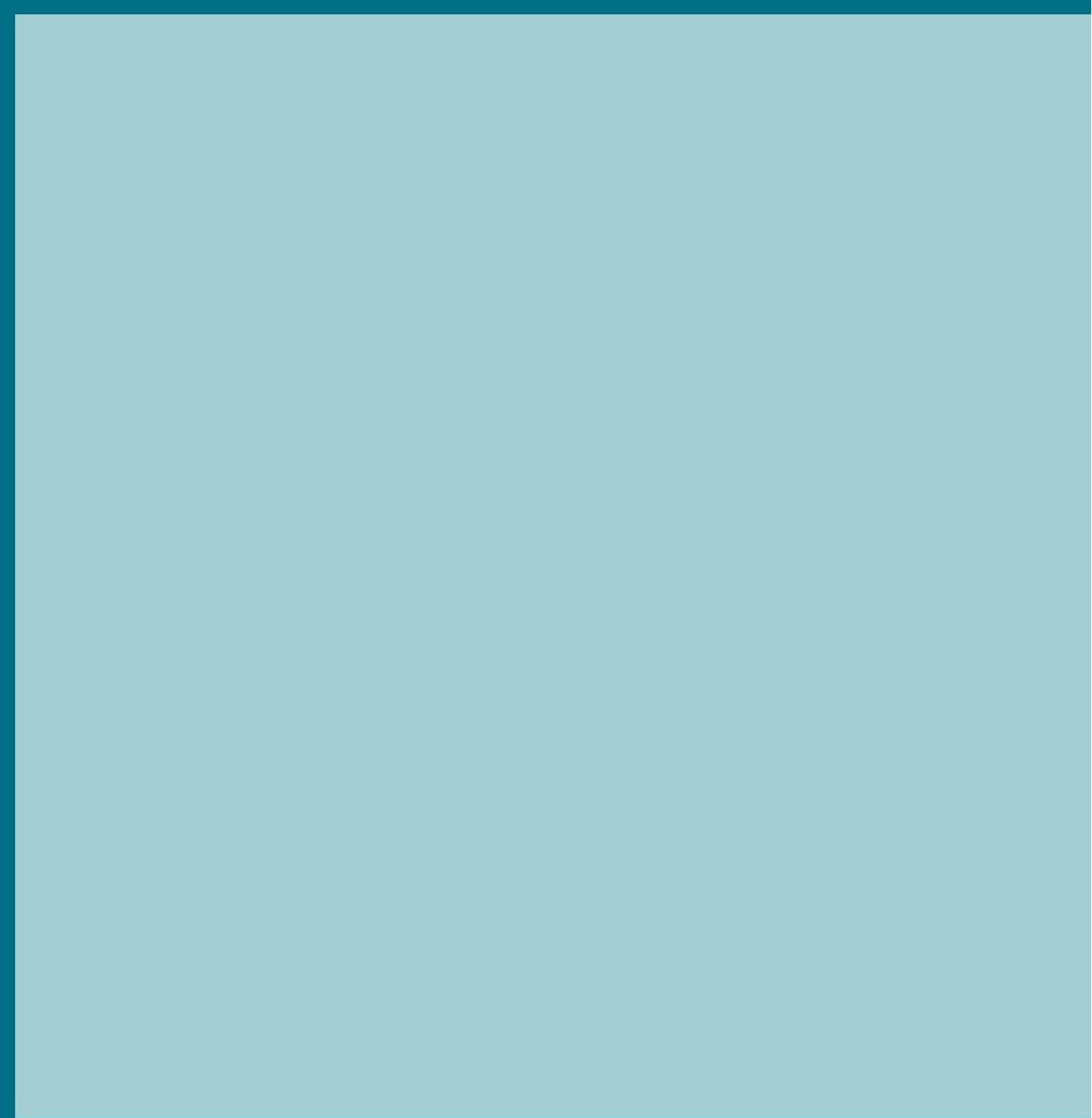

\section{Demandeurs d'asile africains à Tel Aviv, 2013}

() Lisa Anteby-Yemini 


\section{LES DEMANDEURS D'ASILE SUBSAHARIENS EN ISRAËL DE NOUVELLES FRONTIÈRES TERRITORIALES, POLITIQUES ET SOCIALES}

par LISA ANTEBY-YEMINI, chargée de recherches, CNRS, IDEMEC, université Aix-Marseille.

Depuis près d'une dizaines d'années, le nombre de demandeurs d'asile originaires d'Afrique subsaharienne augmente en Israël. Pour la plupart érythréens ou soudanais, ces migrants ont risqué leur vie en passant par le Sinaï égyptien.

L'État hébreu a entrepris de clôturer hermétiquement sa frontière afin d'enrayer ce flux de réfugiés. Les migrants déjà présents en Israël peinent à obtenir un statut dans ce pays qui ne dispose pas de politique d'asile. Leur intégration, difficile, se fait peu à peu avec l'aide de la société civile israélienne.

\section{La traversée des frontières israéliennes}

Depuis 2005, un nombre croissant de demandeurs d'asile, principalement d'Érythrée, du Soudan (Darfour, monts Nouba etrégion de Khartoum) etduSudSoudan, en majorité chrétiens mais aussi musulmans, traversent clandestinement la frontière israélo-égyptienne; ils sont aujourd'hui près de 55000 en Israël, dont la majorité vivent munis d'une protection temporaire dans différentes villes, et dont environ 2000 sont détenus dans un centre de rétention. C'est ainsi qu'apparaît une nouvelle figure de l'Autre, présenté comme un "infiltrateur" par les autorités et comme un "réfugié" par la société civile. La sécurisation de la frontière géographique (avec la construction d'une "barrière de sécurité") et le durcissement des fron- tières politiques et juridiques pour contrer ce flux migratoire donnent parallèlement lieu à une insertion économique, sociale et urbaine de ces nouveaux "résidents" subsahariens.

Il s'agit d'examiner, dans le contexte israélien, la construction progressive de frontières territoriales et de barrières sociales et politiques face à l'essor récent de ces migrations forcées en provenance d'Afrique.

Les logiques à la fois sécuritaire et humanitaire de ces différentes frontières montrent comment les processus de criminalisation, de racialisation et d'exclusion envers les demandeurs d'asile africains vont de pair avec des mécanismes d'appartenance et d'inclusion citoyennes ${ }^{1}$. 


\section{Réseaux de passeurs et trafic de migrants}

Dès le début 2000, les demandeurs d'asile subsahariens empruntent la route vers Israël pour fuir des persécutions religieuses ou ethniques (Sud-Soudanais, Érythréens), un génocide (Soudanais du Darfour), des conflits généralisés (Ivoiriens et Congolais), des violations des droits de l'homme ou la conscription obligatoire illimitée (Érythréens). Ils quittent, de même, les pays de transit, tels la Lybie ou l'Égypte, où ils n'ont généralement pas de permis de travail, ne peuvent scolariser leurs enfants, ne bénéficient d'aucun droit social ou médical, et surtout vivent un racisme quotidien tout en craignant d'être arrêtés, détenus ou même expulsés ${ }^{2}$. Franchir la frontière israélienne représente donc pour ces migrants forcés d'abord la traversée d'une frontière politique, le

Dès le début 2000,

les demandeurs d'asile

subsahariens empruntent

la route vers Israël pour fuir des persécutions religieuses ou ethniques (Sud-Soudanais,

Érythréens), un génocide

(Soudanais du Darfour), des conflits généralisés (Ivoiriens et Congolais), des violations des droits de l'homme ou la conscription obligatoire illimitée (Érythréens).

vant. Entre 2005 et 2009, la plupart des migrants forcés avaient vécu plusieurs années en Égypte, en Libye ou au Soudan, et c'est au Caire qu'ils rencontraient les passeurs bédouins qui les conduisaient à travers le désert du Sinaï jusqu'à la frontière israélienne, pour une somme qui allait de 500 à 3000 dollars environ. Certains décidaient à l'avance de tenter le passage vers Israël, alors que d'autres choisissaient cette destination au cours de leur route. Ils craignaient d'être arrêtés par les Égyptiens avant de franchir la frontière, risquant la prison ou même le rapatriement dans leur pays ou encore d'être harcelés ou abusés sexuellement par les passeurs bédouins, blessés par les barbelés de la frontière, ou pire tués par les tirs des soldats égyptiens ${ }^{3}$. Récemment pourtant, les itinéraires se complexifient et des réseaux extrêmement organisés mènent les migrants directement de leur pays d'origine au Sinaï, en les "revendant" à différents passeurs ; d'autres sont, depuis 2011, kidnappés à la frontière soudano-érythréenne, alors qu'ils n'avaient aucun projet de migrer en Israël. Ils sont détenus au Sinaï où les trafiquants bédouins réclament des rançons allant jusqu'à 30000 dollars, tandis qu'ils sont torturés, utilisés comme "esclaves sexuels" ou "ouvriers forcés", et menacés par le trafic d’organes. Certains ont même été assassinés quand leur rançon restait impayée. Les passeurs contactent par téléphone portable les familles des migrants en Europe, aux Amériques, en Arabie saoudite, en Israël ou en Érythrée et leur font entendre les voix des "otages" qui supplient d'envoyer la somme requise. Les femmes sont d'autant plus vulnérables qu'elles subissent des viols répétés et réclament souvent des avortements quand elles parviennent en Israël. Selon divers rapports, on estime que 4000 individus ont disparu ou péri dans le Sinaï depuis $2007^{4}$.

Quand bien même les migrants se disent informés par ceux parvenus en Israël qu'ils ou elles courent ces risques, beaucoup déclarent ne pas avoir le choix de migrer ailleurs et continuent de tenter la traversée, comme Sarah, une jeune Érythréenne arrivée en Israël en 2011 : "Nous ne sommes pas venus pour travailler; nous sommes venus pour trouver refuge; tout ce que nous voulons, c'est une protection." Face à cette tragédie humaine, les ÉtatsUnis et l'Union européenne appellent à enrayer cette traite d'êtres humains. Dans le même temps, 
ces filières d'“entrepreneurs de la frontière" développent, comme ailleurs, une économie de la migration irrégulière avec des chaînes globales de trafiquants sur des sites multiples ${ }^{5}$. Leurs activités se criminalisent sur le modèle des mafias, d'abord par le rançonnage qui leur permet d'assurer les revenus de cette "industrie" de la migration malgré la baisse du nombre de migrants désirant migrer en Israël, ensuite par le financement d'organisations islamistes qui leur assurent un pouvoir politique dans la région.

\section{Fermeture de la frontière et logique de clôture}

Si les passeurs contrôlent le passage clandestin en deçà de la frontière, ce sont les États-nations qui contrôlent l'entrée ou la sortie du territoire. L'Égypte fait preuve d'une force parfois brutale envers les Subsahariens qui se dirigent vers Israël, suivant la consigne de ne pas les laisser quitter le territoire égyptien. Du côté d'Israël, la gestion de cette frontière a évolué en l'espace de quelques années d'une frontière poreuse à une clôture quasi hermétique.

En effet, pour l'État d'Israël, la traversée clandestine de sa frontière sud par les migrants demandeurs d'asile transforme pour la première fois cet espace en une frontière migratoire avec l'Afrique. C'est pourquoi la construction par Israël d'une barrière pour contrer la migration africaine coïncide désormais avec un nouvel enjeu géostratégique et une logique sécuritaire face à l'instabilité dans la péninsule du Sinaï et à la possible infiltration de groupes terroristes. Auparavant, la frontière israélo-égyptienne n'était délimitée que par des fils barbelés peu élevés et relativement faciles à franchir, comparée à d'autres frontières dotées de moyens de détection et de surveillance sophistiqués. Cependant, de par le nombre croissant de migrants subsahariens qui la franchissent clandes- tinement depuis 2005 (voir tableau ci-dessous), Israël a décidé de construire sur toute sa longueur une barrière frontalière (ou "clôture intelligente", comme l'appellent les officiels israéliens).

À la fin de l'été 2013, un grillage métallique haut de 5 mètres est érigé sur 240 des 266 kilomètres de frontière, surmonté de barbelés et parsemé de tours de surveillance, de radars et de caméras infrarouges. Si le nombre de migrants entrant par la frontière Sud a certes chuté (35 en 2013 contre près de 10000 en 2012), l'instabilité politique au Sinaï et le durcissement du traitement des migrants par les passeurs bédouins et par les autorités israéliennes expliquent aussi ce déclin.

\section{Nombre de migrants ayant franchi la frontière Sud d'Israël (jusqu'en juillet 2013).}

\begin{tabular}{|c|r|}
\hline $\mathbf{2 0 0 6}$ & $\mathbf{2 7 5 8}$ \\
\hline $\mathbf{2 0 0 7}$ & 5132 \\
\hline $\mathbf{2 0 0 8}$ & 8866 \\
\hline $\mathbf{2 0 0 9}$ & 5261 \\
\hline $\mathbf{2 0 1 0}$ & $\mathbf{1 4 7 1 5}$ \\
\hline $\mathbf{2 0 1 1}$ & $\mathbf{1 4 7 1 5}$ \\
\hline $\mathbf{2 0 1 2}$ & 10421 \\
\hline $\mathbf{2 0 1 3}$ & 35 \\
\hline Total & $\mathbf{5 4 2 0 1}$ \\
\hline
\end{tabular}

Au total, 64509 migrants sont entrés clandestinement sur le territoire et 54201 y sont encore en juillet 2013 -dont $25 \%$ en provenance du Soudan et $67 \%$ d'Érythrée.

Source : Israeli Population, Immigration and Border Crossing Authority (Url : http://piba.gov.il/PublicationandTender/ForeignWorkersStat/ Documents/560843nnew4.pdf)

Bien que la construction de cette clôture de sécurité soit un acte unilatéral d'Israël, l'Égypte n'a montré aucune opposition, et aucun désaccord n'existe sur le tracé de cette frontière.

Cette sécurisation reflète lévolution mondiale vers la construction de clôtures physiques et de 
fermeture de l'espace national pour lutter contre l'immigration irrégulière, les trafics illicites et le terrorisme. Cependant, cette barrière soulève de nouveaux dilemmes éthiques et juridiques pour l'État israélien confronté, d'une part,

Depuis 2007, un centre

de rétention, proche de la frontière et attenant à

la prison de Ktziot, détient près de 2000 "infiltrateurs" subsahariens.

\section{à la nécessité de contenir une} migration africaine non juive de demandeurs d'asile et, de l'autre, au devoir de respecter la Convention de Genève sur le statut des réfugiés dont il est signataire. Face à des demandeurs d'asile à présent devant le grillage de la clôture, Israël doit faire le choix entre les laisser entrer, en vertu d'une obligation morale et internationale, ou refuser d'être responsable de migrants devant ses frontières. En septembre 2012, une vingtaine de demandeurs d'asile érythréens ont demandé de passer et seuls deux femmes et un enfant ont pu traverser pour raisons humanitaires.

Enfin, ces dispositifs de contrôle de la souveraineté territoriale ont des implications au niveau des relations internationales, à commencer avec l'Égypte, qui permet à Israël l'externalisation de ses frontières dans ce pays de transit, sans accords formels en matière de migration. C'est aussi en raison de ses relations géostratégiques avec le Sud-Soudan qu'Israël a pu négocier le rapatriement de migrants de cette région. Pour les Subsahariens inexpulsables, la recherche d'un "pays d'accueil tiers" se négocie actuellement avec divers États en échange d'une assistance financière ou militaire.

\section{"Réfugiés africains", mobilisation associative et assistance humanitaire}

Durant l'été 2007, l'afflux massif de demandeurs d'asile subsahariens vers Israël a suscité une mobilisation citoyenne exceptionnelle pour assister ces "réfugiés" perçus comme victimes de persécutions et survivants de génocides, notamment au Darfour. Des bénévoles les hébergent, leur apportent nourriture et vêtements, des étudiants collectent des dons et un climat d'accueil humanitaire entoure ces premiers arrivants. Parce que aucune assistance n'est procurée par le gouvernement, des ONG locales et des associations de demandeurs d'asile (tel African Refugees Development Center) se mettent en place pour offrir les soins de première nécessité (abris d'urgence, dons alimentaires), ainsi que des formations linguistiques (hébreu, anglais) ou professionnelles, un soutien psycho-social (ASSAF) ou des consultations médicales gratuites (Physicians for Human Rights-Israel).

Si la société civile et ces ONG jouent un rôle prépondérant dans la gestion des demandeurs d'asile, elles façonnent aussi l'image de cet Autre subsaharien en Israël. Elles invoquent, tout comme les migrants eux-mêmes, le devoir moral et religieux d'Israël d'accueillir ces "réfugiés africains" (le terme employé) qui recherchent un asile - à l'image des juifs durant la Seconde Guerre mondiale - et qui ont connu des atrocités comparables à la Shoah ${ }^{6}$. Cette rhétorique humanitaire produit la figure de réfugiés vulnérables, privés de droits, victimes de persécutions dans leur pays, de mauvais traitements dans les pays de transit et de trafic d'êtres humains dans le Sinaï, qui méritent l'empathie d'une population israélienne elle-même constituée de réfugiés. Cette précarité devrait justifier de leur accorder l'asile ainsi que des droits sociaux; comme le souligne Didier Fassin dans un autre contexte, considérer les migrants comme proches et socialement acceptables, dans une logique de "politique de compassion", étend les frontières de leur légitimité ${ }^{7}$.

La société civile, par le biais de ces ONG, s'efforce aussi d'étirer les limites des frontières morales et humanitaires en veillant au respect des droits des réfugiés, notamment ceux placés en détention, ou lors de "retours à chaud" vers l'Égypte à l'encontre du principe de non-refoulement. De nombreux 
recours en justice et des assistances juridiques gratuites sont assurés par les avocats d'organisations comme Hotline for Migrant Workers et Anu Plitim ("Nous sommes des réfugiés") pour améliorer la législation en faveur des migrants.

\section{"Infiltrateurs africains", mesures sécuritaires et "illégalisation" des migrants}

À cause de leur entrée irrégulière par la frontière terrestre depuis l'Égypte, les demandeurs d'asile sont généralement repérés par les soldats israéliens qui patrouillent dans cette zone et menés à la base militaire la plus proche, où sont traités les blessés, s'il y a lieu. Ils sont ensuite transférés aux services pénitentiaires censés détenir tout individu entré par "infiltration" (histanenout), à savoir un ressortissant d'un pays ennemi ou ayant traversé un tel pays et entré illégalement sur le territoire. Depuis 2007, un centre de rétention, proche de la frontière et attenant à la prison de Ktziot, détient près de 2000 "infiltrateurs" subsahariens. Leur photo, leurs empreintes digitales et d'autres détails d'identification sont entrés dans une base de données biométriques. La majorité d'entre eux, s'ils étaient originaires de certains pays et après examen médical, étaient jusqu'en juin 2012 "libérés sous condition" après quelques semaines. En revanche, ceux provenant d'autres pays et dont la demande d'asile est jugée infondée, sont menacés d'expulsion. Un véritable dispositif de détention administrative a été ainsi mis en place (tribunaux, juges, interprètes) pour gérer ces migrants, y compris des femmes et des enfants. Le centre de rétention représente donc une seconde frontière à franchir, en quelque sorte, où, comme dans les camps pour étrangers ailleurs, ce tri entraîne l'expulsion ou l'admission" ${ }^{8}$.
Pour faire face à cet afflux sans précédent, Israël octroie à tous les nationaux soudanais et érythréens une protection collective pour motifs humanitaires, sur simple identification comme ressortissant de ces pays, et un titre de séjour renouvelable sans permis de travail ; les migrants du Congo$\mathrm{RDC}$ jouissent de même d'une protection prima facie. Ces statuts n'offrent pas de droits sociaux ni médicaux. De plus, l'accès à la procédure de détermination du statut de réfugié (RSD) ne leur est pas autorisé. Ces formes de protections temporaires signifient que les migrants vivent en situation de "déportabilité", avec la possibilité d'être expulsés à tout moment si les visas de séjour ne sont plus renouvelés (comme pour les Ivoiriens

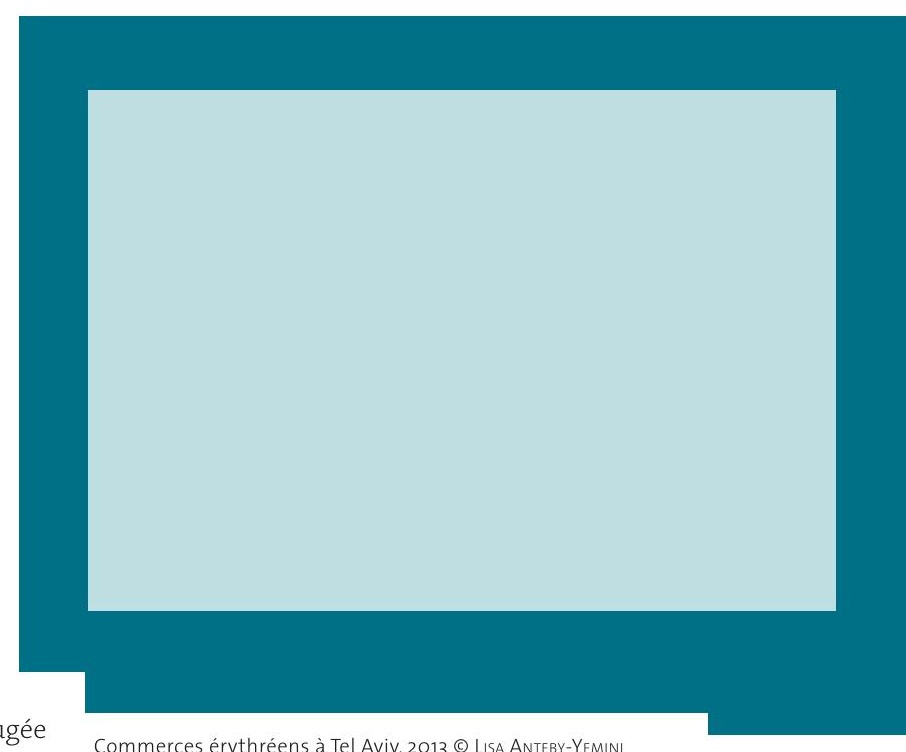

Commerces érythréens à Tel Aviv, 2013 @ LISA ANTEBY-YEMINI

depuis février 2012 et les Sud-Soudanais depuis juin 2012). Seule une mesure - exceptionnelle - en 2007 a octroyé à 500 demandeurs d'asile du Darfour, en tant que "survivants de génocide", le statut de résident temporaire. En créant ces statuts, le gouvernement érige des frontières politiques et 
juridiques selon la nationalité ou la date d'arrivée dans le pays, et entre ces catégories de migrants et les nationaux.

On assiste de plus à un durcissement de la frontière biopolitique par le biais de politiques répressives, telle l'application depuis juin 2012 de l'amendement à la loi de prévention contre l'infiltration, qui pénalise tout "infiltrateur" par la détention systématique jusqu'à trois ans sans jugement ou pour une durée indéfinie s'il vient d'un pays ennemi, tel que le Soudan ${ }^{10}$. Ces mesures sécuritaires, accompagnées d'un discours officiel qualifiant les Subsahariens d'“infiltrateurs" menaçant le pays, participent aussi à ces processus de criminalisation et d'illégalisation" qui brouillent les frontières entre demandeurs d'asile et criminels, entre étrangers avec un statut de protection temporaire et sans-papiers ${ }^{11}$, d'autant plus que la vaste majorité sont juridiquement autorisés à séjourner en Israël puisqu'ils sont enregistrés avec des documents de "libération conditionnelle" auprès du ministère israélien de l'Intérieur... C'est pourquoi la gouvernance de cette migration d'asile est désormais placée sous le signe de la fermeture de la frontière géographique, de l'enfermement des migrants entrant à présent sur le territoire et du renforcement des frontières politico-juridiques de droits, à l'image d'autres pays où la sécurisation des frontières extérieures s'accompagne d'une consolidation des frontières intérieures ${ }^{12}$.

\section{Altérité, racialisation et exclusion des demandeurs d'asile africains}

Les demandeurs d'asile subsahariens, chrétiens ou musulmans, apparaissent, certes, comme une nouvelle figure de l'Autre dans la société israélienne. Mais, au-delà de cette altérité religieuse et culturelle, ce sont les frontières sociales qui margina- lisent ces migrants sans statut juridique ni droits, condamnés pour certains à vivre dans des parcs publics et à survivre de dons alimentaires d'ONG ou d'initiatives privées - faute d'infrastructures gouvernementales d'accueil -, confrontés à l'exclusion urbaine et exploités sur le marché du travail. Bien qu'une circulaire du ministère du Travail et de l'Industrie assure que les employeurs de demandeurs d'asile (sans permis de travail) ne seront pas sanctionnés, les employeurs profitent de leur statut précaire en les embauchant à la journée ou à la semaine et en ne respectant pas le droit du travail. Les migrants sont employés dans l'hôtellerie, la restauration, le nettoyage, le gardiennage, les travaux de construction, de voierie et sur les marchés des grandes villes ; ils sont souvent endettés envers leurs proches (ils doivent rembourser leur migration et parfois leur rançon) et survivent donc dans un état de grande pauvreté.

Certaines municipalités essaient d'exclure les demandeurs d'asile subsahariens de l'espace urbain en érigeant des frontières à l'accès au logement ou aux services éducatifs. Sur les murs du quartier de Shapira, à Tel Aviv, on apercevait à l'hiver 2010 des affiches signées de rabbins demandant de ne plus louer d'appartements aux migrants africains, à côté de publicités pour un concert érythréen ou un nouveau restaurant soudanais. En décembre 2012, des banderoles aux fenêtres affichaient "Rentrez au Soudan", "Retournez chez vous"... Une rhétorique qui dépeint cette migration comme une menace démographique pour l'identité nationale juive alimente ces sentiments antimigratoires. Pour le Premier ministre Benyamin Netanyahou, cette migration est une "catastrophe nationale, une menace pour notre société, notre économie et notre sécuritét ${ }^{13}$. Les discours de certains membres de la Knesset sont particulièrement virulents, parlant d' infiltrateurs" qui répandent des maladies, commettent des crimes et représentent "un cancer dans notre corps ${ }^{14}$. 
Les habitants des quartiers sud de Tel Aviv, où se concentrent plus de 40000 demandeurs d'asile subsahariens, se plaignent de l'insécurité et de problèmes de criminalité. Des comités pour la défense des quartiers se forment et des manifestations de résidents ont lieu, comme celle organisée dans le quartier Hatikva, en décembre 2010, avec pour slogan : "Arrêtez la peur dans les quartiers, renvoyez les infiltrateurs chez eux." Un rabbin y déclare : "On a assez souffert; ils ont conquis notre quartier ; c'est insoutenable que mes enfants ne puissent plus sortir et que les Soudanais aient tous les droits." Ces discours montrent à quel point la présence massive de cette minorité visible est perçue comme une conquête spatiale et urbaine, qui fait appel à des métaphores de frontières envahies et de quartiers assiégés. Depuis 2012, des actes criminels commis par des Israéliens contre des demandeurs d'asile africains se multiplient : cocktails molotov lancés contre leurs jardins d'enfants informels, appartements incendiés, commerces vandalisés et agressions. Par ces processus de distanciation des migrants subsahariens comme ultimes "Autres", se jouent les revendications territoriales des citoyens pour défendre les frontières "nationales" à travers des frontières urbaines, sociales et spatiales.

\section{Appartenance urbaine et inclusion citoyenne}

Face à ces mécanismes de rejet, les demandeurs d'asile, ne pouvant ni être expulsés ni poursuivre leur route migratoire, se sédentarisent dans les agglomérations israéliennes, travaillent dans le secteur des services, construisent des lieux de sociabilité et deviennent des acteurs urbains de facto. Ils donnent des prénoms israéliens à leurs enfants et les scolarisent dans les écoles publiques (en vertu d'une loi de scolarisation obligatoire
- quel que soit le statut légal de l'enfant) qui les initient aux fêtes juives et à la culture israélienne ; ils accrochent des drapeaux israéliens à leur balcon, commencent à parler hébreu, pratiquent des loisirs (sport, plage), établissent des églises et des groupes de prière musulmans et se politisent pour exiger davantage de droits.

Sibeaucoup restent sans emploi ou ne travaillent que partiellement, d'autres ont su profiter d'opportunités économiques et réussissent désormais à soutenir financièrement leurs Les habitants des quartiers sud de Tel Aviv, où se concentrent plus de 40 ooo demandeurs d'asile subsahariens, se plaignent de l'insécurité et de problèmes de criminalité. familles dans les pays d'origine ou de transit et à rembourser leurs dettes. Certains connaissent même une ascension économique et, par le biais de l'entreprenariat ethnique, ouvrent, surtout à Tel Aviv, des restaurants, des cybercafés, des bars, des boîtes de nuit, des salons de coiffure, des services de photos (baptêmes, mariages), des salles de fête, des "cinémas" improvisés et des magasins de téléphonie et d'informatique ${ }^{15}$. C'est aussi par leurs relations de travail que certains tissent des liens amicaux avec des Israéliens qui les invitent chez eux, les assistent dans certaines démarches ou défendent leur cause.

Tout en vivant aux marges de la société, les demandeurs d'asile subsahariens contribuent à l'économie urbaine (demande sur le marché de location immobilière ; consommation de produits alimentaires, d'appareils de téléphonie ou informatique ; usage de transports publics, transferts de fonds), de même que dans d'autres villes globales où réfugiés et migrants sont consommateurs de biens et de services ${ }^{16}$. Par ailleurs, la reterritorialisation des cultures soudanaise et érythréenne dans les villes israéliennes crée de nouveaux lieux diasporiques et de nouveaux paysages ethniques (ethnoscapes) qui participent à la mondialisation de l'espace urbain 
et à la pluralité religieuse du pays, déjà amorcées par les migrations de travail asiatique, sud-américaine et africaine des années $1990^{17}$.

Enfin, les demandeurs d'asile se mobilisent pour revendiquer des droits sociaux en tant que "réfugiés", mais aussi en tant que "citoyens urbains". Aussi leurs demandes de "droits urbains" attenants à une "résidence sociale" (social residency) pressentelles les autorités de les reconnaître malgré leur exclusion. Ces processus d'insertion économique, scolaire, sociale et urbaine leur permettent de développer de nouvelles formes d'appartenance qui défient les frontières de la citoyenneté.

\section{Conclusion}

La construction d'une barrière sur la frontière sud d'Israël, les nouvelles mesures sécuritaires et le climat d'hostilité envers les Subsahariens vont vers un renforcement des frontières physiques et symboliques dans la société et l'espace israéliens, découlant de logiques sécuritaires et nationalistes. Simultanément, des processus d'inclusion et des formes d'appartenance permettent aussi à la société civile et aux demandeurs d'asile de contourner ces frontières internes en suivant des logiques humanitaires.

Si la frontière physique se referme avec l'achèvement de la clôture, il s'agit de savoir si les demandeurs d'asile subsahariens parviendront à franchir les frontières politiques, économiques et sociales de la société ou s'ils demeureront en Israël bloqués dans cette "sédentarité forcée", dans une zone frontalière qu'ils ne peuvent traverser ni en migrant ailleurs, ni en retournant au Soudan ou en Érythrée.

De ce point de vue, cette migration d'asile exige que le gouvernement développe une législation sur l'asile (qui n'existe pas) et des statuts de résidence temporaire dans un pays qui est en passe de devenir une nouvelle terre de transit et une frontière supplémentaire de l'Europe, comme d'autres pays de la rive sud de la Méditerranée. 